Mater. Res. Soc. Symp. Proc. Vol. 1319 @ 2011 Materials Research Society

DOI: 10.1557/opl.2011.740

\title{
Reconstructing the past to prevent future loss: The dyeing industry of Pompeii
}

\section{Hopkins H. J.}

School of Engineering, Design and Technology, University of Bradford, Bradford, West Yorkshire, BD7 1DP, United Kingdom.

\begin{abstract}
The industrial dyeing apparatus excavated in Pompeii have been preserved and remain in situ. To understand Pompeii's economy, and its place in the Roman world, it is necessary to first understand the capabilities of a single industry. Before this study, the size of the dyeing industry was calculated by applying theory to a superficial measurement of the remains. This study was the first to realise that to understand an industry it was necessary to reconstruct and use the relevant parts.

The most comprehensive survey of the apparatus was undertaken. A full-scale physical replica was constructed from materials that physically and thermally matched the originals. This study was the first to define the dyeing cycle time, temperatures reached and fuel type and quantity required. Finite Element Analysis (FEA) was used to model a virtual replica to show the effect of external influences on the materials during use. The lead metal data did not exist before this study. This was the first use of FEA to model an archaeological apparatus or artefact of more than one material. The dyeing industry had been far smaller than originally thought.

Archaeological virtual replications tend to be aesthetic. This study produced a rare physical replication. When this is combined with data from the original survey and physical replicas each apparatus is now 'preserved by record' and may be recreated. Some of the apparatus in Pompeii have been amended in an attempt to reconstruct and preserve them. This study has shown that the amendments are incorrect and potentially misleading.

Prior to this study the size of the industry was a controversial 'unanswerable' question. This study provided a solid foundation that answered the question and illustrated a new approach, through a method that provided a means of preserving the apparatus for the future.
\end{abstract}

\section{INTRODUCTION}

The city of Pompeii was simultaneously destroyed and preserved by a pyroclastic volcanic event in AD79. As it was encased as a 'time capsule' the city survived as a whole, so it is now possible to study industrial workshops and equipment within the wider context of the city that they supplied. Wider economic questions can be examined as it may be assumed that the number of workshops, in particular the apparatus contained within them, would be the number required for the supply of a city (unless there was a dependence on imports). Dyeing apparatus and dyeing workshops are unusual as they can be unambiguously identified. Prior to the volcanic eruption, Pompeii had been destroyed and rebuilt following an earthquake in AD62. The long term effects included the movement of industry within the city: manufacturing workshops were constructed in buildings that were formerly of residential use. Although the location and architectural style of the workshops changed, the dyeing apparatus itself remained the same in design.

Once the size and scale of one manufacturing industry has been reconstructed, it is possible to apply this throughout the city to gain a better understanding of the economy in which the 
industry operated. Following this it is then possible to place the importance of Pompeii's economy within the wider Roman world. Before the current study, all previous understanding of the dyeing apparatus from Pompeii was based on a theoretical understanding of a superficial measurement of the remains. No single dyeing apparatus survives intact and so the standard design was actually an untested composite. A single survey had been undertaken by Moeller in $1976^{(1)}$, but there was no practical understanding of how the apparatus operated so it was not possible to determine if they had been altered. Moeller was criticised at the time as "going further than the evidence could take him"(2) and subsequent authors disagreed with his findings

(3) (4), but the theoretical understanding of the dyeing industry based on only unexplored surface evidence formed the basis for all subsequent calculations of the size, significance and scale of the industry before the current study. The current study was the first to realise that the only way in which it was possible to understand the operating parameters and the physical capabilities of a dyeing apparatus was to physically reconstruct one and use it. Use of the physical replica led to unforeseen questions, the exploration of which led to unanticipated consequences.

\section{THEORY}

In 2002 a dyeing apparatus was reconstructed from data collected in Pompeii ${ }^{(5)}$ (Fig. One).

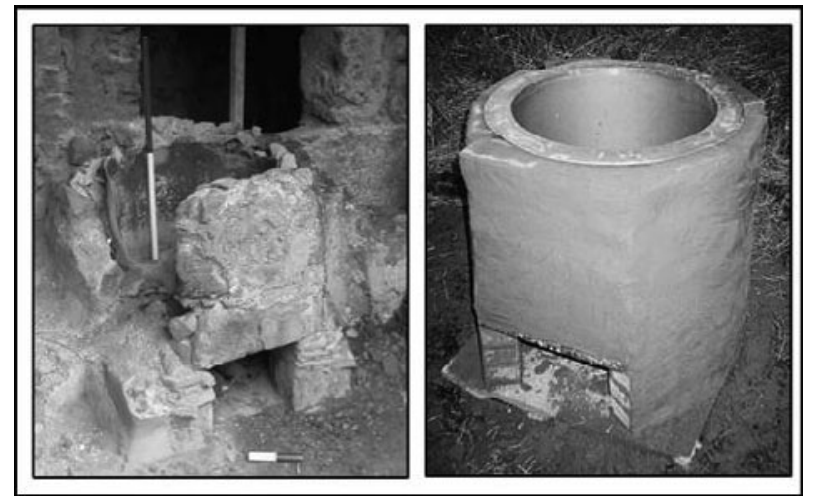

Figure One. Apparatus 3 in property I viii 19 and the replica apparatus

It was found that there was sufficient oxygen for the complete combustion of pine wood but that charcoal would not burn. Each dyeing cycle required $8 \mathrm{~kg}$ of pine, 90 litres of water, and the dyeing cycle took 24 hours. An ergonomic understanding of the apparatus could be made and 'average Romans' (male and female) were found who could undertake it. Most importantly, the viability of the design was tested ${ }^{(6)}(7)$. This formed the basis for the most comprehensive survey of the Pompeii apparatus and properties to be undertaken ${ }^{(7)}{ }^{(8)}$. The record of each apparatus was the most detailed yet made. Colour photographs were taken of each apparatus and of each group and workshop. Sketches and written descriptions were made. Measurements were taken of each. A plan of each workshop was drawn. The design of each apparatus was ergonomically assessed 
and assessed for viability as a dyeing apparatus. An understanding of how the apparatus operated meant that it was possible to discern what was missing from each apparatus, what had been added or amended and whether any previous restoration had been sympathetic. One apparatus was discovered to have been mis-identified in Moeller's original survey and so was discounted. This understanding and the record of each apparatus formed the foundation for a new understanding of the dyeing industry and a calculation of the size and scale of manufacture that is grounded in what is physically possible, rather than theoretical assumptions.

Following the survey it was noted that there were two basic designs of apparatus: flued and unflued (Fig. Two).
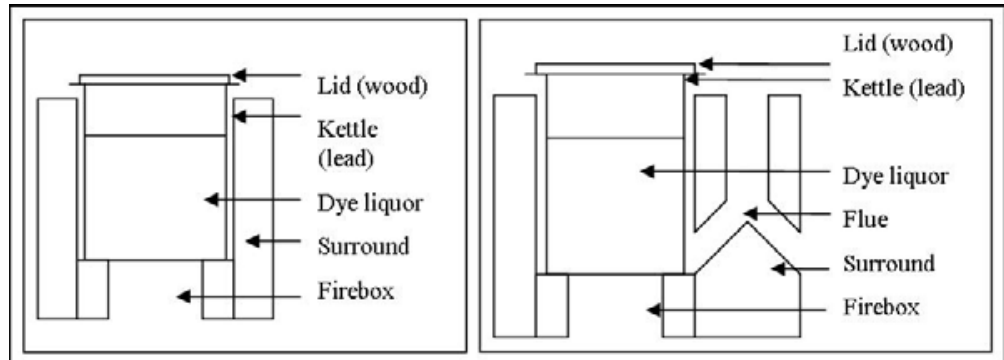

Figure Two. Schematic of the two apparatus designs: with flue and without flue.

The replica apparatus was amended to explore the difference that adding a flue would make (Fig. Three).

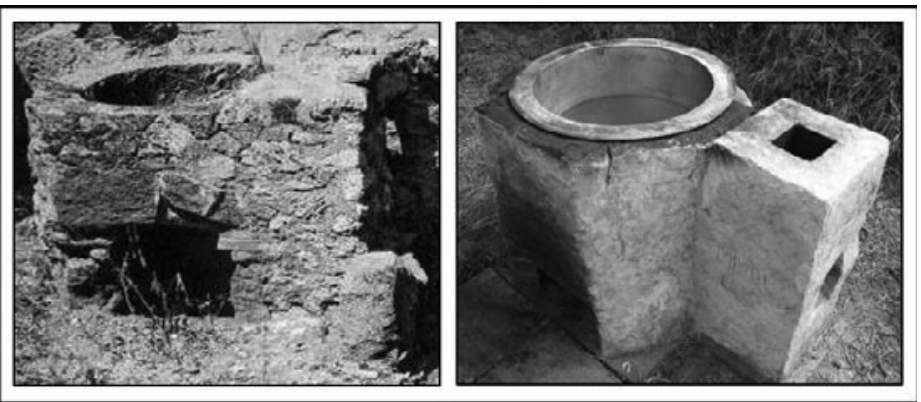

Figure Three. Apparatus 5 in property VII xiv 17 and the replica, both with flue.

It was concluded that although the fire was easier to control with a flue present, the difference overall was not substantial, but as the flued apparatus were in enclosed workshops it was probably that the flues were required to assist in drawing air through the firebox. It was 
understood at the time of the survey that although it was necessary to record the details that were seen as relevant at that time, as the study might develop in an unknown direction it could be possible that further 'irrelevant' details may be required at a later date. This means that the record was as complete as possible.

The concentric rings at the base of the remaining metal kettles had been recorded as an archaeological curiosity, but further than that no significance had been attached to them ${ }^{(7)}$ (Fig. Four).

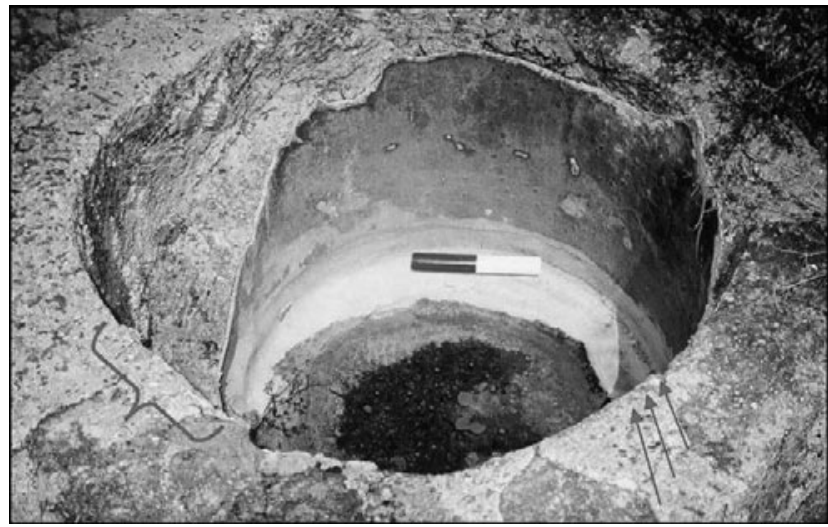

Figure Four. Lead kettle in apparatus 5, property VII xiv 17.

Left: kettle has been cemented into place after it was warped in the eruption.

Right: arrows indicating the concentric circles at the base of the kettle caused by creep.

It was realised that they were the evidence of 'creep', time-dependent strain acting on the lead kettle ${ }^{(9)}$. If the creep was quick-acting it could cause a failure of the kettle which in turn would cause a loss in time, money and productivity. As this would limit the output of the dyeing industry it was necessary to understand how this occurred. It has been argued that to construct a replica from different materials to an original is not a true reconstruction ${ }^{(10)}$. But modern Health and Safety requirements meant that it was not possible to use the same materials and so instead physical substitutes had to be made. Brick and building mortar replaced the rubble and corrosive lime mortar, stainless steel replaced lead. Each of these replacement materials matched the physical and thermal properties of the original, which as the aim of the reconstruction was to test the viability of design and to determine the time taken for heating and cooling was all that was required. A problem now arose as it was not possible to study the effect of creep using the replica apparatus as stainless steel and lead creep at different rates. As the length of time taken for the creep to occur was unknown, even if using a lead kettle had been possible it was not practicable to construct a replica and wait.

This problem was addressed by the construction and use of a virtual replica. The survey had provided sufficient details that there were enough data to construct a virtual replica that matched the originals in Pompeii and the physical reconstruction. Moving into the virtual world brought 
other advantages: an apparatus would be in a controlled environment, time could be slowed or speeded up, the design or materials of the apparatus could be changed. The exact point at which failure occurred could be slowed to the point that the mechanism of failure could be studied in minute detail. Virtual reconstruction of an archaeological artefact of more than one material, or that was to be heated and cooled, or was an industrial apparatus rather than a single product had not been tried before. The data used to simulate the lead had to be derived first hand ${ }^{(7)}$. The apparatus was reconstructed and examined through the use of Finite Element Analysis (FEA). The materials and design of the apparatus were deconstructed into discrete parts, 'finite elements', each one represented by an equation (Fig. Five). As a force or temperature was applied, its effect could be seen as it passed through the material ${ }^{(11)}$. The computer program used was ABAQUS as this specialises in change caused by heat transfer ${ }^{(12)}$. The exact process of breaking down the artefact to allow its input into the computer program has been discussed in detail elsewhere $^{(7)(13)}$.

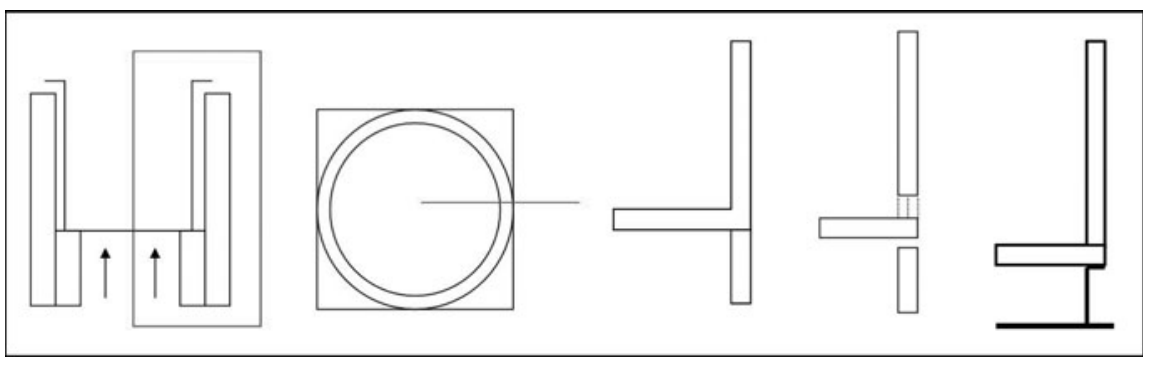

Figure Five. Schematic showing the break down of the apparatus for input into the Finite Element Analysis program. The metal kettle, brick support and floor were replicated. As the apparatus was symmetrical, it was only necessary to construct half. The kettle was formed from two sheets joined (a cylinder to a disc). The water within the kettle was simulated using hydrostatic pressure.

It was discovered that the kettle was subject to creep, but that it did not fail, even after six months of use, which was unforeseen (Fig. Six).

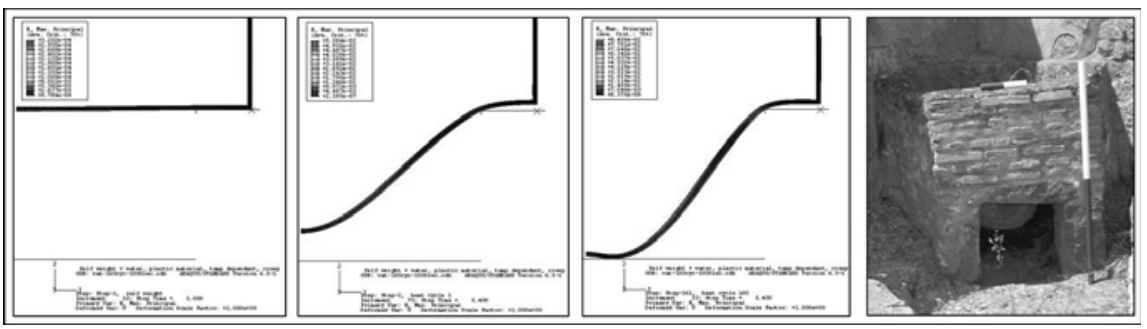

Figure Six. Virtual simulation of the dyeing apparatus, showing the strain. Left: apparatus under load, at $20^{\circ} \mathrm{C}$. Second from left: apparatus after one complete thermal cycle. Third from left: apparatus after the equivalent of six months use. Note the kettle is resting on the floor but has not failed. Right: photograph of apparatus 6 in property VII xiv 17 showing creep. 
This meant that it was now possible to calculate the size and scale of the dyeing industry. It has been calculated that between 48, $972-53,106$ fleeces were dyed annually and if the population was 12,000 this would be the equivalent of $4.1-4.4$ fleeces or $8.16-8.84 \mathrm{~kg}$ annually per person. In practical terms that is the equivalent of a large washing machine load per person, per year. It is possible that the majority of clothing was not dyed, but as soft furnishings were, it is possible to see how, after the allowance for the cloth in a temple or other public building was subtracted, that would be a very small amount per person ${ }^{(7)}$.

It was thought that the use of FEA could be beneficial in the future because it allowed a quicker and easier method of reconstructing the apparatus and viewing each in use. There are thirty-five apparatus altogether and each differs slightly in dimensions, design and materials. It was foreseen that it would now be possible to construct each of the apparatus individually and to alter them to explore their properties. They could be reconstructed within a virtual workshop to allow the accumulation of heat or exhaust gases to be studied and to see if there would have been sufficient ventilation for complete combustion to take place within each apparatus if used simultaneously. The fuel requirement could be better understood, which in turn would allow the storage requirement to be calculated and the place of fuel and other consumables within the wider economy to be understood. FEA would allow an understanding of the minutiae that would allow a greater understanding of the dyeing industry as a whole.

Overall, virtual reconstructions in the archaeological world concentrate on the aesthetics of the artefact and its environment. This allows the public to understand a site in three dimensions and to move within it, something that can be difficult to envisage from sketches and descriptions or an incomplete object. One example is of the reconstruction of an artefact and its placing within its original context, subject to the original lighting conditions ${ }^{(14)}$. Without this virtual reconstruction it would not be possible to explore the significance of this positioning or its effects. This study differs as the aesthetic is not considered; instead the physical properties are what are of importance. The apparatus will respond as an original physical apparatus responded. This means that theoretically if a virtual aesthetic replica was heated and cooled, no change could occur, whereas if a virtual physical replica was heated and cooled, it would warp and its change through use could be observed and understood.

There was an unforeseen advantageous consequence of the approach of this study and its diversification into virtual reconstruction. When building work is undertaken in the United Kingdom, any archaeology on the site that is not deemed important enough for 'preservation in situ' is instead 'preserved by record'. This means that it is excavated and recorded prior to development work and so while it is physically lost, it survives in the photographs and records that were taken at the time. The aim of the survey of the dyeing apparatus was to record each apparatus to allow interpretation and from this an understanding of the size of the industry. But by completing the survey this study has also created a record of each of the apparatus that will continue to exist should the unthinkable happen and the apparatus become destroyed.

The survey was undertaken in 2002 . Had the survey been undertaken significantly earlier it would have missed the start of the digital age. The timing of the survey means that it is now possible to combine field records with an application of FEA and computer modelling to not only provide a complete photographic and descriptive record, but the reconstruction of each apparatus to create a virtual record. As the approach of this study was not to create an aesthetic replica but a replica that has the same physical properties and so responds the same as the original, it is now possible to 'preserve by record' not just the appearance of an apparatus, in two dimensions or three dimensions, but the actual apparatus in full. A model can be created of each 
apparatus allowing an archaeologist to examine not just how it looks but how it reacts to external applications such as temperature or force. A virtual reconstruction can be used to test the reliability of the remains that have been found and recorded, long after the original artefact has been lost. With this greater understanding it is possible to preserve and restore remains more sympathetically, leaving a far more accurate picture of the artefact to archaeologists of the future. Furthermore, it can be used for experiments to understand how an apparatus reacted within the wider environment that it had been constructed and operated in. Like the model in the current study, this means that it is possible to not just use the apparatus without damaging the original, but also to examine the effects when the original has been lost. Should new data come to light, the virtual model can be amended and updated.

\section{DISCUSSION}

This approach became pertinent recently due to events in Pompeii that were unforeseen at the time of the survey. The remains in Pompeii are degrading quickly and so some have been restored. Some previous consolidation of remains is now indistinguishable from the original structures built in antiquity. This has caused misinterpretation that was only revealed following an ergonomic examination of the apparatus (Fig. Seven).

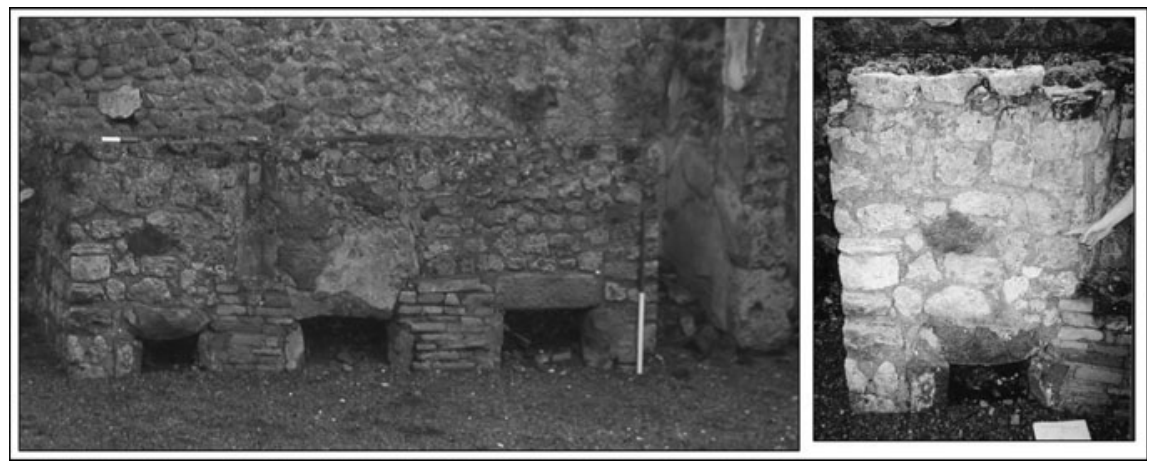

Figure Seven. Apparatus 1, 2, 3, property IX iii 2 . The present height was originally deemed accurate. Ergonomic assessment showed this to be unlikely. Closer inspection showed a line in the mortar where the height had been increased, indicated here by a finger.

To determine what is original, what is false and what is inaccurate, it is necessary to have an understanding of how the original apparatus would have worked. The physical reconstruction, the survey and the computer model together allowed the physical properties and operation of the apparatus to be understood. This allowed not just the remains to be assessed, but an assessment of how each of the apparatus was designed, constructed, how it had been restored and the affect that this would have on calculating the size and significance of the industry. It was then possible to perceive inaccuracies and remove them from the calculations. 
Recently an attempt has been made at consolidation and restoration, but due to a lack of understanding of the design, construction or operating of the apparatus the work has gone beyond this and caused far greater damage. Apparatus 7 and 8 in property Vi4 have been covered with cement and dyeing kettles have been sealed into the top of each apparatus (Fig. Eight). The restoration is not only inaccurate, but has obliterated details of the original remains and it is not now possible to study the originals.

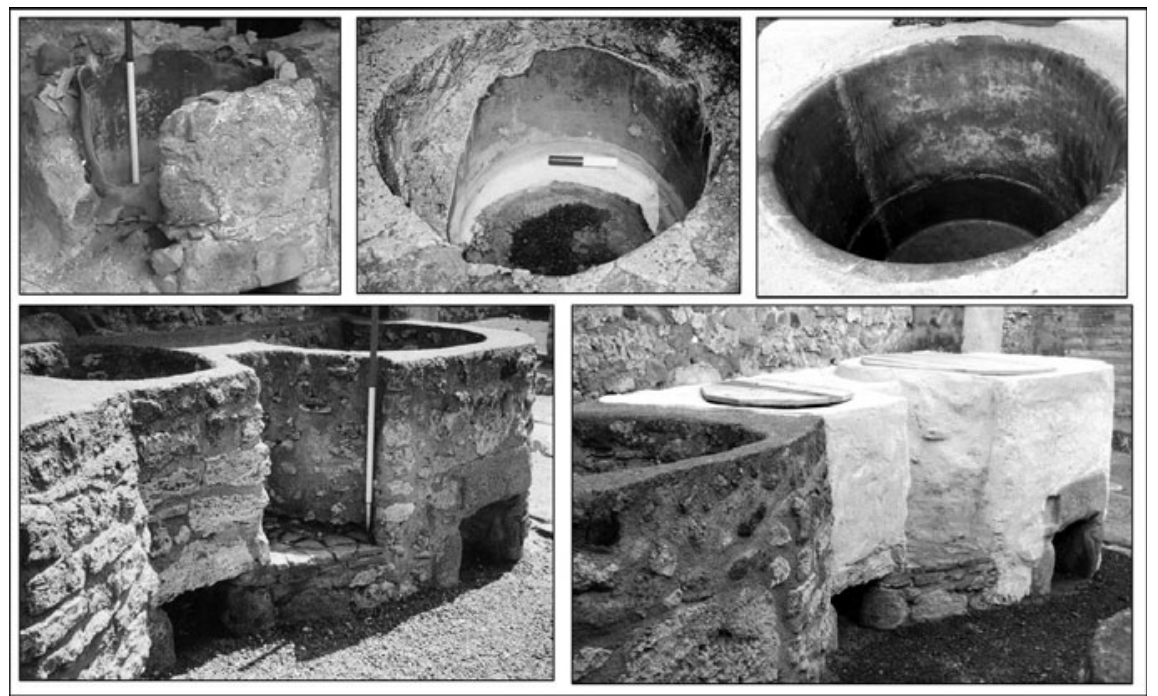

Figure Eight. The 'restoration' of apparatus 7 and 8 in property Vi5. Top left: apparatus 3 in property I viii 19, showing that the kettle was not cemented into place. Top middle: apparatus 5 in property VII xiv 17, showing the kettle was cemented into place after the eruption. Top right: the restored apparatus in Vi5, showing the kettle inaccurately cemented into place.

Bottom left: apparatus 7 and 8 in property Vi5 in 2002. Note: there has been light restoration, but the apparatus features are discernable. Bottom right: apparatus following 'restoration' in 2010. Photograph, bottom right, by Sera Baker of University of Nottingham, 2010.

The survey and use of the physical apparatus demonstrated that the reconstruction of apparatus 7 and 8 is not an accurate representation of how the apparatus were originally constructed. The lead kettles were originally free-standing: they were not cemented into the base of the apparatus, or on to the walls and they were not sealed in with cement at the top (Fig. One, Fig. Eight, top left). Apparatus 3 in property I viii 19, has split longitudinally, giving the clearest example in Pompeii of a 'cross-section' of a dyeing apparatus. It is possible to confirm that the kettle was not cemented into the apparatus but rested on the base. It would have been held firm by its own weight and the weight of the water it contained. A small air-gap existed between the kettle and the wall of the surround. The only kettle to have been cemented into place was apparatus 5 in property VII xiv 17. This kettle is warped and has been cemented into place in its warped state. 
This means that it was cemented into place after it had warped during the eruption (Fig. Four, Fig. Eight). It may be concluded that the kettles were not cemented into place and to do so is inaccurate.

The importance of this is not just aesthetic. These inaccuracies change the design of the apparatus and how the apparatus functions. The small gap between the kettle and the apparatus acted as an exhaust, drawing air up through the apparatus and so increasing airflow through the firebox. An increase in airflow would increase combustion. This air heated the kettle as it rose. By cementing the kettle into place in the restoration, the airflow and heat transfer within the apparatus would be altered. This would produce an inaccurate understanding of the consumables and time required for the apparatus to operate. All economic understanding based on this would be wrong. Over time the inaccurate restoration will break down and it will be possible to see the original apparatus, but by the time that this occurs it will not be possible to discern between the original and the restoration, as has been demonstrated with apparatus 1, 2, 3 in property IX iii 2 (Fig. Seven). The use of the physical replica and the use of the virtual replica allowed an understanding of how the apparatus operated. This allowed an accurate record to be made during the survey. As there is a record of the apparatus and the ability to construct a virtual physical replica, it is still possible to examine the apparatus as if such damage had not occurred.

\section{CONCLUSIONS}

Construction of an apparatus through virtual methods allows a greater understanding of its physical properties, how it operated and its place within a wider context, in this example the size and scale of the dyeing industry of Pompeii based on an understanding of the dyeing apparatus. This in turn allows better preservation of the original artefact. But there is a further advantage to virtual replication: it allows the preservation of apparatus by record and their recreation should the original become lost. Pompeii is still beneath Vesuvius. One day it will erupt again and bury the city. When that day comes it will be possible to recreate not just an aesthetic version in which an archaeologist may walk around, but a virtual physical version in which an archaeologist can truly understand the artefacts that are preserved for posterity through virtual replication.

Post Script: On $7^{\text {th }}$ November 2010, as this article was being drafted, 'The House of the Gladiators' collapsed in Pompeii. It had previously been restored and although it was not open to the public it was not thought to be at risk ${ }^{(15)}$. This graphically illustrates the urgent need for the recording of fragile remains that are degrading quickly and the suddenness with which the original can be lost. The technology exists to both record what remains and to reconstruct it. If virtually reconstructed it can be accessible throughout the world.

\section{ACKNOWLEDGMENTS}

I would like to thank Sera Baker of Nottingham University for kindly providing me with the photographs of the restored apparatus and permitting me to use them in this publication.

\section{REFERENCES}

1. W. Moeller, The wool trade of ancient Pompeii. (E.J.Bril:Leiden, 1976). 
2. J. P. Wild, Textile History. 8, 180 (1977).

3. W. Jongman, The Economy and Society of Pompeii. (J.C. Gieben, Amsterdam. 1988).

4. R. Laurence, Roman Pompeii Space and Society. (Routledge, London. 1994)

5. R. Janaway and D. Robinson, unpublished survey of dyeing apparatus in Pompeii, 1994.

6. H. Hopkins, L. Willimott, R. Janaway, D. Robinson, W. Seale. Understanding the economic influence of the dyeing industry in Pompeii through the application of experimental archaeology and thermodynamics. In Scientific Analysis of Ancient and Historic Textiles, Informing Preservation, Display and interpretation eds R. C. Janaway and P. Wyeth. (Archetype Publications, London. 2005).

7. H. J. Hopkins An investigation of the parameters that would influence the scale of the dyeing industry in Pompeii An application of experimental archaeology and computer simulation techniques to investigate the scale of manufacture of the dyeing industry and the factors that influence output. (PhD thesis, University of Bradford, 2007).

8. H. J. Hopkins. Papers of the British School of Rome. Gazetteer of the dyeing works of Pompeii. forthcoming

9. P. Greenfield, Creep of Metals of high temperatures. (Mills and Boon, 1972)

10. J. Coles, Archaeology by Experiment. (Hutchinson University Library, London. 1973).

11. M. J. Fagan, Finite Element Analysis: Theory and Practice. (Longman Scientific \& Technical: Harlow, England. 1992)

12. ABAQUS 6.3 /Standard User's Manual, Vol II, (Hibbitt, Karlsson and Sorensen Inc. 2002).

13. H. J. Hopkins. Archaeometry. Creep of Lead Dyeing Vessels in Pompeii. forthcoming

14. J. Happa, M. Mudge, K. Debattista, A. Artusi, A. Gonçalves, A. Chalmers, Virtual Reality, 14:155-182 (2010).

15. http://www.bbc.co.uk/news/world-europe-11704720 viewed 7th November 2010. This is a link from the main page of BBC news, United Kingdom. Responsibility for upkeep of this site does not rest with the author. 